\title{
Construction of quasi-twisted codes and enumeration of defining polynomials
}

Research Article

\author{
T. Aaron Gulliver, Vadlamudi Ch. Venkaiah
}

Abstract: Let $d_{q}(n, k)$ be the maximum possible minimum Hamming distance of a linear $[n, k]$ code over $\mathbb{F}_{q}$. Tables of best known linear codes exist for small fields and some results are known for larger fields. Quasi-twisted codes are constructed using $m \times m$ twistulant matrices and many of these are the best known codes. In this paper, the number of $m \times m$ twistulant matrices over $\mathbb{F}_{q}$ is enumerated and linear codes over $\mathbb{F}_{17}$ and $\mathbb{F}_{19}$ are constructed for $k$ up to 5 .

2010 MSC: 94B05, 94B65, 94A55

Keywords: Finite fields, Twistulant matrices, Quasi-twisted codes, Optimal codes, Griesmer bound

\section{Introduction}

Let $\mathbb{F}_{q}$ denote the finite field of $q$ elements, and $V(n, q)$ the vector space of $n$-tuples over $\mathbb{F}_{q}$. A linear $[n, k]$ code $C$ of length $n$ and dimension $k$ over $\mathbb{F}_{q}$ is a $k$-dimensional subspace of $V(n, q)$. The elements of $C$ are called codewords. The (Hamming) weight of a codeword is the number of non-zero coordinates, and the minimum distance of $C$ is the smallest weight among all non-zero codewords of $C$. An $[n, k, d]$ code is an $[n, k]$ code with minimum distance $d$. Let $A_{i}$ be the number of codewords of weight $i$ in $C$. Then the numbers $A_{0}, A_{1}, \ldots, A_{n}$ are called the weight distribution of $C$.

A central problem in coding theory is that of optimizing one of the parameters $n, k$ and $d$ for given values of the other two. One can find $d_{q}(n, k)$, the largest value of $d$ for which there exists an $[n, k, d]$ code over $\mathbb{F}_{q}$, or $n_{q}(k, d)$, the smallest value of $n$ for which there exists an $[n, k, d]$ code over $\mathbb{F}_{q}$. A code which achieves either of these values is called optimal. Tables of best known linear codes exist for $q=2$ to $9[6], 11[3]$ and 13 [4].

T. Aaron Gulliver (Corresponding Author); Department of Electrical and Computer Engineering, University of Victoria, PO Box 1700, STN CSC, Victoria, BC V8W 2Y2, Canada (email: agullive@ece.uvic.ca).

Vadlamudi Ch. Venkaiah; School of Computer and Information Sciences, University of Hyderabad, Gachibowli, Hyderabad 500 046, India (email:venkaiah@hotmail.com,vvcs@uohyd.ernet.in) 
The Griesmer bound is a well-known lower bound on $n_{q}(k, d)$

$$
n_{q}(k, d) \geq g_{q}(k, d)=\sum_{j=0}^{k-1}\left\lceil\frac{d}{q^{j}}\right\rceil,
$$

where $\lceil x\rceil$ denotes the smallest integer $\geq x$. For $k \leq 2$, there exist codes that attain equality in the Griesmer bound for all $q$ and $d$. The Singleton bound [12] is a lower bound on $n_{q}(k, d)$ and is given by

$$
n_{q}(k, d) \geq d+k-1 \text {. }
$$

Codes that meet this bound are called maximum distance separable (MDS). MDS codes exist for all values of $n \leq q+1$. Thus, for $q=17 \mathrm{MDS}$ codes exist for all lengths 18 or less, and for $q=19$ all lengths 20 or less. Note that all MDS codes are optimal. For larger lengths and dimensions, far less is known about codes over $\mathbb{F}_{17}$ and $\mathbb{F}_{19}$.

MDS self-dual codes $(k=n / 2)$, of lengths $2,4,6,8,10$ and 18 over $\mathbb{F}_{17}$ are known [1], as well as self-dual $[12,6,6],[14,7,7],[16,8,8],[20,10,10],[22,11,10]$ and $[24,12,10]$ codes. The $[14,7,8]$ and $[20,10,10]$ extended quadratic residue (QR) codes are given in [13]. Using Magma [2], it was determined that the next extended QR code has parameters [44,22,18]. MDS self-dual codes of lengths 4, 8, 12 and 20 over $\mathbb{F}_{19}$ are known [1], as well as self-dual $[16,8,8]$ and $[24,12,9]$ codes. The $[18,9,9]$ and $[32,16,14]$ extended QR codes are given in [13]. In this paper, codes over $\mathbb{F}_{17}$ and $\mathbb{F}_{19}$ for $k$ up to 5 are presented. These codes establish lower bounds on the minimum distance. Many of these meet the Singleton and/or Griesmer bounds, and so are optimal.

\section{Quasi-twisted codes}

A constacyclic shift of an $m$-tuple

$$
\left(x_{0}, x_{1}, \ldots, x_{m-1}\right)
$$

is the $m$-tuple

$$
\left(\lambda x_{m-1}, x_{0}, \ldots, x_{m-2}\right)
$$

where $\lambda \in \mathbb{F}_{q} \backslash\{0\}$, and a constacyclic shift by $p$ positions is the $m$-tuple

$$
\left(\lambda x_{m-p}, \ldots, \lambda x_{m-1}, x_{0}, \ldots, x_{m-p-1}\right) .
$$

A linear code $C$ is said to be quasi-twisted (QT) if a constacyclic shift of any codeword by $p$ positions is also a codeword in $C$ [8]. Note that quasi-twisted codes generalize the classes of constacyclic codes $(p=1)$, quasi-cyclic codes $(\lambda=1)$, cyclic codes $(\lambda=1, p=1)$, and negacyclic codes $(\lambda=-1, p=1)$. The length of a QT code considered here is $n=m p$. With a suitable permutation of coordinates, many QT codes can be characterized in terms of $m \times m$ twistulant matrices. In this case, a QT code can be transformed into an equivalent code with generator matrix

$$
G=\left[\begin{array}{lllll}
B_{0} & B_{1} & B_{2} & \ldots & B_{p-1}
\end{array}\right]
$$

where $B_{i}, i=0,1, \ldots, p-1$, is an $m \times m$ twistulant matrix (also known as a constacyclic matrix), over $\mathbb{F}_{q}$ of the form [9]

$$
B=\left[\begin{array}{ccccc}
b_{0} & b_{1} & b_{2} & \cdots & b_{m-1} \\
\lambda b_{m-1} & b_{0} & b_{1} & \cdots & b_{m-2} \\
\lambda b_{m-2} & \lambda b_{m-1} & b_{0} & \cdots & b_{m-3} \\
\vdots & \vdots & \vdots & & \vdots \\
\lambda b_{1} & \lambda b_{2} & \lambda b_{3} & \cdots & b_{0}
\end{array}\right]
$$


where $\lambda \in \mathbb{F}_{q} \backslash\{0\}$ and $b_{i}, 0 \leq i \leq m-1$, are elements of $\mathbb{F}_{q}$. When $\lambda=1$, a twistulant matrix is a circulant matrix, and when $\lambda=-1$, a twistulant matrix is known as a negacirculant matrix [8].

The algebra of $m \times m$ twistulant matrices over $\mathbb{F}_{q}$ is isomorphic to the algebra of polynomials in the $\operatorname{ring} \mathbb{F}_{q}[x] /\left(x^{m}-\lambda\right)$ if $B_{i}$ is mapped onto the polynomial $b_{i}(x)=b_{0, i}+b_{1, i} x+b_{2, i} x^{2}+\ldots+b_{m-1, i} x^{m-1}$ formed from the entries in the first row of $B_{i}$. The $b_{i}(x)$ associated with a QT code are called the defining polynomials [7]. The set $\left\{b_{0}(x), b_{1}(x), \ldots, b_{p-1}(x)\right\}$ defines an $[m p, m]$ QT code with $k \leq m$.

\section{Defining polynomials}

The construction of QT codes requires a representative set of defining polynomials. These are the equivalence class representatives of a partition of the set of polynomials of degree less than $m$. For defining polynomials, multiplication by a non-zero element of $\mathbb{F}_{q}$ does not change the weight and hence does not change the equivalence class. Thus, two polynomials $r_{j}(x)$ and $r_{i}(x)$ are said to be equivalent if

$$
r_{j}(x)=\gamma x^{l} r_{i}(x) \bmod \left(x^{m}-\lambda\right)
$$

for some integer $l \geq 0$ and scalar $\gamma \in \mathbb{F}_{q} \backslash\{0\}$.

A closed-form expression for the number of defining polynomials is now given. Let $g$ be the permutation $(1,2, \ldots, m)$ so that $g$ maps $i$ to $i+1$, for $1 \leq i \leq m-1$, and $m$ to 1 . Therefore, $g^{i}, 1 \leq i \leq m$, is also a permutation and has order $\frac{m}{\operatorname{gcd}(m, i)}$ in the symmetric group of degree $m$. Thus, the action of $g$ on the $m$-tuple $x=\left(x_{1}, x_{2}, \ldots, x_{m}\right)$ changes $x$ to $\left(x_{m}, x_{1}, x_{2}, \ldots, x_{m-1}\right)$ where $x_{i} \in \mathbb{F}_{q}$. Now let $\lambda g$ be such that the action of $\lambda g$ on the $m$-tuple changes $x$ to $\left(\lambda x_{m}, x_{1}, x_{2}, \ldots, x_{m-1}\right)$, the action of $(\lambda g)^{2}$ on $x$ results in $\left(\lambda x_{m-1}, \lambda x_{n}, x_{1}, x_{2}, \ldots, x_{m-2}\right)$, and similarly for other powers. Then the order of $\lambda g$ is $\operatorname{Ord}(\lambda) m$, where $\operatorname{Ord}(\lambda)$ is the order of $\lambda$ in $\mathbb{F}_{q}$. Further, let $t(\lambda g), t \in \mathbb{F}_{q} \backslash\{0\}$, be such that it changes $x$ to $\left(t \lambda x_{m}, t x_{1}, t x_{2}, \ldots, t x_{m-1}\right)$. The action of $t(\lambda g)^{2}$ changes $x$ to $\left(t \lambda x_{m-1}, t \lambda x_{m}, t x_{1}, t x_{2}, \ldots, t x_{m-2}\right)$, and similarly for other powers. The equivalence relation is induced by the action of the group consisting of the elements $t(\lambda g)^{i}, 1 \leq i \leq \operatorname{Ord}(\lambda) m, t \in \mathbb{F}_{q} \backslash\{0\}$. Distinct equivalence classes correspond to distinct orbits under the action of this group and so can be enumerated using Burnside's Lemma [5, 9].

Definition 3.1. An ordered $m$-tuple (or word of length $m$ ), $x=\left(x_{1}, x_{2}, \ldots, x_{m}\right)$, is said to be fixed by $t(\lambda g)^{i}, t, \lambda \in \mathbb{F}_{q} \backslash\{0\}, 1 \leq i \leq \operatorname{Ord}(\lambda) m$, if the $m$-tuple $x$ remains unchanged by the action of $t(\lambda g)^{i}$.

Theorem 3.2. The number of words of length $m$ over the alphabet $\mathbb{F}_{q}$ fixed by $t(\lambda g)^{i}$ for some fixed $\lambda \in \mathbb{F}_{q} \backslash\{0\}, t \in \mathbb{F}_{q} \backslash\{0\}, 1 \leq i \leq \operatorname{Ord}(\lambda) m$, is $q^{\operatorname{gcd}(m, i)}$ if $t^{\left(\frac{m}{\operatorname{gcd}(m, i)}\right)} \lambda^{\left(\frac{i}{\operatorname{gcd}(m, i)}\right)}=1$. Otherwise, it is 1 .

Proof. Let $x=\left(x_{1}, x_{2}, \ldots, x_{m}\right)$ be a word of length $m$ over $\mathbb{F}_{q}$. Then the relation between the components of $x$ before and after the action of $t(\lambda g)^{i}$ is

$$
\begin{aligned}
x_{1} & =t \lambda x_{m-i+1} \\
x_{2} & =t \lambda x_{m-i+2} \\
x_{3} & =t \lambda x_{m-i+3} \\
\vdots & \\
x_{i-1} & =t \lambda x_{m-1} \\
x_{i} & =t \lambda x_{m} \\
x_{i+1} & =t x_{1} \\
x_{i+2} & =t x_{2} \\
\vdots & \\
x_{m-1} & =t x_{m-i-1} \\
x_{m} & =t x_{m-i}
\end{aligned}
$$


if $1 \leq i \leq m$. If $m+1 \leq i \leq 2 m$, the relation between the components of $x$ is

$$
\begin{aligned}
x_{1} & =t \lambda^{2} x_{m-i+1} \\
x_{2} & =t \lambda^{2} x_{m-i+2} \\
x_{3} & =t \lambda^{2} x_{m-i+3} \\
\vdots & \\
x_{i-1} & =t \lambda^{2} x_{m-1} \\
x_{i} & =t \lambda^{2} x_{m} \\
x_{i+1} & =t \lambda x_{1} \\
x_{i+2} & =t \lambda x_{2} \\
\vdots & \\
x_{m-1} & =t \lambda x_{m-i-1} \\
x_{m} & =t \lambda x_{m-i} .
\end{aligned}
$$

Thus in general, the relation between the components of $x$ is

$$
\begin{aligned}
x_{1} & =t \lambda^{j} x_{m-i+1} \\
x_{2} & =t \lambda^{j} x_{m-i+2} \\
x_{3} & =t \lambda^{j} x_{m-i+3} \\
\vdots & \\
x_{i-1} & =t \lambda^{j} x_{m-1} \\
x_{i} & =t \lambda^{j} x_{m} \\
x_{i+1} & =t \lambda^{j-1} x_{1} \\
x_{i+2} & =t \lambda^{j-1} x_{2} \\
\vdots & \\
x_{m-1} & =t \lambda^{j-1} x_{m-i-1} \\
x & =t \lambda^{j-1} x_{m-i}
\end{aligned}
$$

if $(j-1) m+1 \leq i \leq j m, 1 \leq j \leq \operatorname{Ord}(\lambda)$.

Let $\operatorname{gcd}(m, i)=h$. Then, from the expressions above, the orbit of $x_{m}$ is

$$
\begin{aligned}
x_{m} & =t x_{m-i}=t^{2} x_{m-2 i}=\cdots=t^{\left\lfloor\frac{m}{i}\right\rfloor} x_{m-\left\lfloor\frac{m}{i}\right\rfloor i} \\
& =t^{\left(\left\lfloor\frac{m}{i}\right\rfloor+1\right)} \lambda x_{2 m-\left(\left\lfloor\frac{m}{i}\right\rfloor+1\right) i}=t^{\left\lfloor\left\lfloor\frac{m}{i}\right\rfloor+2\right)} \lambda x_{2 m-\left(\left\lfloor\frac{m}{i}\right\rfloor+2\right) i} \\
& =\cdots=t^{\left(\left\lfloor\frac{2 m}{i}\right\rfloor-1\right)} \lambda x_{2 m-\left(\left\lfloor\frac{2 m}{i}\right\rfloor-1\right) i} \\
& =t^{\left(\left\lfloor\frac{2 m}{i}\right\rfloor\right)} \lambda x_{2 m-\left(\left\lfloor\frac{2 m}{i}\right\rfloor\right) i}=t^{\left(\left\lfloor\frac{2 m}{i}\right\rfloor+1\right)} \lambda^{2} x_{3 m-\left(\left\lfloor\frac{2 m}{i}\right\rfloor+1\right) i} \\
& =t^{\left(\left\lfloor\frac{2 m}{i}\right\rfloor+2\right)} \lambda^{2} x_{3 m-\left(\left\lfloor\frac{2 m}{i}\right\rfloor+2\right) i}=\cdots=t^{\left(\left\lfloor\frac{3 m}{i}\right\rfloor-1\right)} \lambda^{2} x_{3 m-\left(\left\lfloor\frac{3 m}{i}\right\rfloor-1\right) i} \\
& =t^{\left(\left\lfloor\frac{3 m}{i}\right\rfloor\right)} \lambda^{2} x_{3 m-\left(\left\lfloor\frac{3 m}{i}\right\rfloor\right) i}=t^{\left(\left\lfloor\frac{3 m}{i}\right\rfloor+1\right)} \lambda^{3} x_{4 m-\left(\left\lfloor\frac{3 m}{i}\right\rfloor+1\right) i} \\
& =t^{\left(\left\lfloor\frac{3 m}{i}\right\rfloor+2\right)} \lambda^{3} x_{4 m-\left(\left\lfloor\frac{3 m}{i}\right\rfloor+2\right) i} \\
& =\cdots=t^{\left(\left\lfloor\frac{4 m}{i}\right\rfloor-1\right)} \lambda^{3} x_{4 m-\left(\left\lfloor\frac{4 m}{i}\right\rfloor-1\right) i} \\
& =t^{\left(\left\lfloor\frac{4 m}{i}\right\rfloor\right)} \lambda^{3} x_{4 m-\left(\left\lfloor\frac{4 m}{i}\right\rfloor\right) i}=\cdots=t^{\left.\left\lfloor\left(\frac{i}{h}-1\right) \frac{m}{i}\right\rfloor+1\right)} \lambda^{\left(\frac{i}{h}-1\right)} x_{\frac{i m}{h}-\left(\left\lfloor\frac{\left(\frac{i}{h}-1\right) m}{i}\right\rfloor+1\right) i} \\
& =t^{\left(\left\lfloor\left(\frac{i}{h}-1\right) \frac{m}{i}\right\rfloor+2\right)} \lambda^{\left(\frac{i}{h}-1\right)} x_{\frac{i m}{h}-\left(\left\lfloor\frac{\left(\frac{i}{h}-1\right) m}{i}\right\rfloor+2\right) i} \\
& =\cdots=t^{\left(\left\lfloor\left(\frac{i}{h} \frac{m}{i}\right)\right\rfloor-1\right)} \lambda^{\left(\frac{i}{h}-1\right)} x_{\frac{i m}{h}-\left(\left\lfloor\frac{i m}{i}\right\rfloor-1\right) i}=t^{\left(\left\lfloor\left(\frac{i}{h} \frac{m}{i}\right)\right\rfloor\right)} \lambda^{\left(\frac{i}{h}\right)} x_{m},
\end{aligned}
$$


and so we also have

$$
\begin{aligned}
x_{m-1} & =t x_{m-1-i}=t^{2} x_{m-1-2 i}=\cdots=t^{\left\lfloor\frac{m}{i}\right\rfloor} x_{m-1-\left\lfloor\frac{m}{i}\right\rfloor i} \\
& =t^{\left(\left\lfloor\frac{m}{i}\right\rfloor+1\right)} \lambda x_{2 m-1-\left(\left\lfloor\frac{m}{i}\right\rfloor+1\right) i}=t^{\left(\left\lfloor\frac{m}{i}\right\rfloor+2\right)} \lambda x_{2 m-1-\left(\left\lfloor\frac{m}{i}\right\rfloor+2\right) i} \\
& =\cdots=t^{\left(\left\lfloor\frac{2 m}{i}\right\rfloor-1\right)} \lambda x_{2 m-1-\left(\left\lfloor\frac{2 m}{i}\right\rfloor-1\right) i}=t^{\left(\left\lfloor\frac{2 m}{i}\right\rfloor\right)} \lambda x_{2 m-1-\left(\left\lfloor\frac{2 m}{i}\right\rfloor\right) i} \\
& =t^{\left(\left\lfloor\frac{2 m}{i}\right\rfloor+1\right)} \lambda^{2} x_{3 m-1-\left(\left\lfloor\frac{2 m}{i}\right\rfloor+1\right) i}=t^{\left(\left\lfloor\frac{2 m}{i}\right\rfloor+2\right)} \lambda^{2} x_{3 m-1-\left(\left\lfloor\frac{2 m}{i}\right\rfloor+2\right) i} \\
& =\cdots=t^{\left(\left\lfloor\frac{3 m}{i}\right\rfloor-1\right)} \lambda^{2} x_{3 m-1-\left(\left\lfloor\frac{3 m}{i}\right\rfloor-1\right) i}=t^{\left(\left\lfloor\frac{3 m}{i}\right\rfloor\right)} \lambda^{2} x_{3 m-1-\left(\left\lfloor\frac{3 m}{i}\right\rfloor\right) i} \\
& =t^{\left(\left\lfloor\frac{3 m}{i}\right\rfloor+1\right)} \lambda^{3} x_{4 m-1-\left(\left\lfloor\frac{3 m}{i}\right\rfloor+1\right) i}=t^{\left(\left\lfloor\frac{3 m}{i}\right\rfloor+2\right)} \lambda^{3} x_{4 m-1-\left(\left\lfloor\frac{3 m}{i}\right\rfloor+2\right) i} \\
& =\cdots=t^{\left(\left\lfloor\frac{4 m}{i}\right\rfloor-1\right)} \lambda^{3} x_{4 m-1-\left(\left\lfloor\frac{4 m}{i}\right\rfloor-1\right) i}=t^{\left(\left\lfloor\frac{4 m}{i}\right\rfloor\right)} \lambda^{3} x_{4 m-1-\left(\left\lfloor\frac{4 m}{i}\right\rfloor\right) i} \\
& =\cdots=t^{\left(\left\lfloor\left(\frac{i}{h}-1\right) \frac{m}{i}\right\rfloor+1\right)} \lambda^{\left(\frac{i}{h}-1\right)} x_{\frac{i m}{h}-1-\left(\left\lfloor\frac{\left(\frac{i}{h}-1\right) m}{i}\right\rfloor+1\right) i} \\
& =t^{\left(\left\lfloor\left(\frac{i}{h}-1\right) \frac{m}{i}\right\rfloor+2\right)} \lambda^{\left(\frac{i}{h}-1\right)} x_{\frac{i m}{h}-1-\left(\left\lfloor\frac{\left(\frac{i}{h}-1\right) m}{i}\right\rfloor+2\right) i} \\
& =\cdots=t^{\left(\left\lfloor\left(\frac{i}{h} \frac{m}{i}\right)\right\rfloor-1\right)} \lambda^{\left(\frac{i}{h}-1\right)} x_{\frac{i m}{h}-1-\left(\left\lfloor\frac{\frac{i m}{h}}{i}\right\rfloor-1\right) i} \\
& =t^{\left(\left\lfloor\left(\frac{i}{h} \frac{m}{i}\right)\right\rfloor\right)} \lambda^{\left(\frac{i}{h}\right)} x_{m-1} .
\end{aligned}
$$

Similar expressions exist for $x_{m-2}, x_{m-3}, \ldots, x_{m-h+2}$, and in general

$$
\begin{aligned}
x_{m-h+1} & =t x_{m-h+1-i}=t^{2} x_{m-h+1-2 i}=\cdots=t^{\left\lfloor\frac{m}{i}\right\rfloor} x_{m-h+1-\left\lfloor\frac{m}{i}\right\rfloor i} \\
& =t^{\left(\left\lfloor\frac{m}{i}\right\rfloor+1\right)} \lambda x_{2 m-+1-\left(\left\lfloor\frac{m}{i}\right\rfloor+1\right) i}=t^{\left(\left\lfloor\frac{m}{i}\right\rfloor+2\right)} \lambda x_{2 m-h+1-\left(\left\lfloor\frac{m}{i}\right\rfloor+2\right) i} \\
& =\cdots=t^{\left(\left\lfloor\frac{2 m}{i}\right\rfloor-1\right)} \lambda x_{2 m-h+1-\left(\left\lfloor\frac{2 m}{i}\right\rfloor-1\right) i}=t^{\left(\left\lfloor\frac{2 m}{i}\right\rfloor\right)} \lambda x_{2 m-h+1-\left(\left\lfloor\frac{2 m}{i}\right\rfloor\right) i} \\
& =t^{\left(\left\lfloor\frac{2 m}{i}\right\rfloor+1\right)} \lambda^{2} x_{3 m-h+1-\left(\left\lfloor\frac{2 m}{i}\right\rfloor+1\right) i}=t^{\left(\left\lfloor\frac{2 m}{i}\right\rfloor+2\right)} \lambda^{2} x_{3 m-h+1-\left(\left\lfloor\frac{2 m}{i}\right\rfloor+2\right) i} \\
& =\cdots=t^{\left(\left\lfloor\frac{3 m}{i}\right\rfloor-1\right)} \lambda^{2} x_{3 m-h+1-\left(\left\lfloor\frac{3 m}{i}\right\rfloor-1\right) i}=t^{\left(\left\lfloor\frac{3 m}{i}\right\rfloor\right)} \lambda^{2} x_{3 m-h+1-\left(\left\lfloor\frac{3 m}{i}\right\rfloor\right) i} \\
& =t^{\left(\left\lfloor\frac{3 m}{i}\right\rfloor+1\right)} \lambda^{3} x_{4 m-h+1-\left(\left\lfloor\frac{3 m}{i}\right\rfloor+1\right) i}=t^{\left(\left\lfloor\frac{3 m}{i}\right\rfloor+2\right)} \lambda^{3} x_{4 m-h+1-\left(\left\lfloor\frac{3 m}{i}\right\rfloor+2\right) i} \\
& =\cdots=t^{\left(\left\lfloor\frac{4 m}{i}\right\rfloor-1\right)} \lambda^{3} x_{4 m-h+1-\left(\left\lfloor\frac{4 m}{i}\right\rfloor-1\right) i}=t^{\left(\left\lfloor\frac{4 m}{i}\right\rfloor\right)} \lambda^{3} x_{4 m-h+1-\left(\left\lfloor\frac{4 m}{i}\right\rfloor\right) i} \\
& =\cdots=t^{\left(\left\lfloor\left(\frac{i}{h}-1\right) \frac{m}{i}\right\rfloor+1\right)} \lambda^{\left(\frac{i}{h}-1\right)} x_{\frac{i m}{h}-h+1-\left(\left\lfloor\frac{\left(\frac{i}{h}-1\right) m}{i}\right\rfloor+1\right) i} \\
& =t^{\left(\left\lfloor\left(\frac{i}{h}-1\right) \frac{m}{i}\right\rfloor+2\right)} \lambda^{\left(\frac{i}{h}-1\right)} x_{\frac{i m}{h}-h+1-\left(\left\lfloor\frac{\left(\frac{i}{h}-1\right) m}{i}\right\rfloor+2\right) i} \\
& =\cdots=t^{\left(\left\lfloor\left(\frac{i}{h} \frac{m}{i}\right)\right\rfloor-1\right)} \lambda^{\left(\frac{i}{h}-1\right)} x_{\frac{i m}{h}-h+1-\left(\frac{i m}{i h}-1\right) i} \\
& =t^{\left(\frac{i}{h} \frac{m}{i}\right)} \lambda^{\left(\frac{i}{h}\right)} x_{m-h+1} .
\end{aligned}
$$

Thus, the orbits of $x_{m}, x_{m-1}, \ldots, x_{m-h+1}$ are fixed by the action of $t(\lambda g)^{i}$ if and only if $t^{\frac{m}{h}} \lambda^{\frac{i}{h}}=$ $t^{\frac{m}{\operatorname{gcd}(m, i)}} \lambda^{\frac{i}{\operatorname{gcd}(m, i)}}=1$. Since there are $h=\operatorname{gcd}(m, i)$ independent orbits and each orbit can take on $q$ values, $q^{h}=q^{\operatorname{gcd}(m, i)}$ words are fixed by $t(\lambda g)^{i}$. If $t^{\frac{m}{\operatorname{gcd}(m, i)}} \lambda^{\frac{i}{\operatorname{gcd}(m, i)}} \neq 1$, then there is only one orbit consisting of all $m$ elements of the word. In this case, since $\lambda \neq 1$, the only word that is fixed is the zero word.

Theorem 3.3. The number of defining polynomials of length $m$ over $\mathbb{F}_{q}$ is

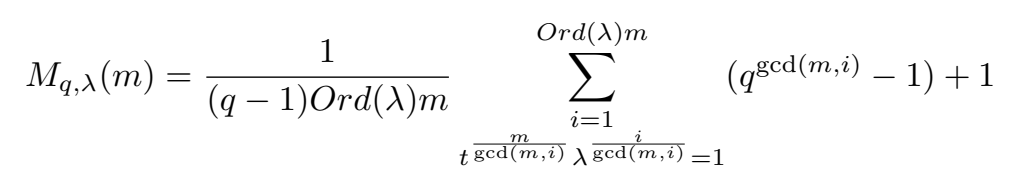


Proof. There are $(q-1) \operatorname{Ord}(\lambda) m$ permutations given by $t(\lambda g)^{i}$. Thus, by Burnside's lemma [5], the number of orbits of words of length $m$ over an alphabet of size $q$ is equal to the average number of words fixed by each $t(\lambda g)^{i}, 1 \leq i \leq \operatorname{Ord}(\lambda) m, t \in \mathbb{F}_{q} \backslash\{0\}$. Therefore, we have

$$
M_{q, \lambda}(m)=\frac{1}{(q-1) \operatorname{Ord}(\lambda) m} \sum_{\substack{i=1 \\ t \in \mathbb{F}_{q} \backslash\{0\}}}^{\operatorname{Ord}(\lambda) m}\left|\operatorname{Fix} t(\lambda g)^{i}\right|,
$$

where $\mid$ Fix $t(\lambda g)^{i} \mid$ denotes the number of words fixed by $t(\lambda g)^{i}$.

From Theorem 1, the number of words fixed by $t(\lambda g)^{i}$ is either $q^{\operatorname{gcd}(m, i)}$ or 1 depending on whether $t^{\frac{m}{\operatorname{gcd}(m, i)}} \lambda^{\frac{i}{\operatorname{gcd}(m, i)}}=1$ or not. Therefore

$$
\begin{aligned}
& M_{q, \lambda}(m)=\frac{1}{(q-1) \operatorname{Ord}(\lambda) m}\left\{\sum_{\substack{i=1 \\
t \overline{\operatorname{gcd}(m, i)} \lambda^{\overline{\operatorname{gcd}(m, i)}}=1}}^{\operatorname{Ord}(\lambda) m} q^{\operatorname{gcd}(m, i)}+\left\{\sum_{\substack{m \\
\frac{m}{\operatorname{gcd}(m, i)} \lambda^{\overline{\operatorname{gcd}(m, i)}} \neq 1}}^{\operatorname{Ord}(\lambda) m} 1\right\}\right. \\
& =\frac{1}{(q-1) \operatorname{Ord}(\lambda) m}\left\{\sum_{\substack{m \\
t \overline{\operatorname{gcd}(m, i)} \lambda \overline{\operatorname{gcd}(m, i)}=1}}^{\operatorname{Ord}(\lambda) m} q^{\operatorname{gcd}(m, i)}\right.
\end{aligned}
$$

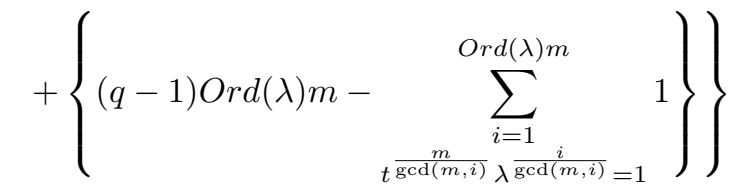

$$
\begin{aligned}
& =\frac{1}{(q-1) \operatorname{Ord}(\lambda) m}\left\{\sum_{\substack{m \\
t^{\overline{g c d}(m, i)} \lambda^{\overline{\operatorname{gcd}(m, i)}}=1}}^{\operatorname{Ord}(\lambda) m}\left(q^{\operatorname{gcd}(m, i)}-1\right)+(q-1) \operatorname{Ord}(\lambda) m\right\} \\
& =\frac{1}{(q-1) \operatorname{Ord}(\lambda) m} \sum_{\substack{i=1 \\
t^{\overline{\operatorname{gcd}}(m, i)} \lambda^{\overline{\operatorname{gcd}(m, i)}}=1}}^{\operatorname{Ord}(\lambda) m}\left(q^{\operatorname{gcd}(m, i)}-1\right)+1
\end{aligned}
$$

Example 3.4. Let $q=13, m=2$, and $\lambda=4$. Since 6 is the least integer such that $\lambda^{6}=4^{6}=1$, we 
have $\operatorname{Ord}(\lambda)=\operatorname{Ord}(4)=6$. Then

$$
\begin{aligned}
M_{13,4}(2)= & \frac{1}{144} \sum_{t \in \mathbb{R}_{13} \backslash\{0\}, t^{\operatorname{gcd}(2, i)}}^{12}\left(13^{\operatorname{gcd}(2, i)}=1\right. \\
= & \frac{1}{144}\left\{\left[\left(13^{\operatorname{gcd}(2,12)}-1\right)\right]+\left[\left(13^{\operatorname{gcd}(2,5)}-1\right)+\left(13^{\operatorname{gcd}(2,11)}-1\right)\right]\right\} \\
& +\frac{1}{144}\left\{\left[\left(13^{\operatorname{gcd}(2,8)}-1\right)\right]+\left[\left(13^{\operatorname{gcd}(2,10)}-1\right)\right]\right\} \\
& +\frac{1}{144}\left\{\left[\left(13^{\operatorname{gcd}(2,3)}-1\right)+\left(13^{\operatorname{gcd}(2,9)}-1\right)\right]+\left[\left(13^{\operatorname{gcd}(2,1)}-1\right)+\left(13^{\operatorname{gcd}(2,7)}-1\right)\right]\right\} \\
& +\frac{1}{144}\left\{\left[\left(13^{\operatorname{gcd}(2,1)}-1\right)+\left(13^{\operatorname{gcd}(2,7)}-1\right)\right]\right\} \\
& +\frac{1}{144}\left\{\left[\left(13^{\operatorname{gcd}(2,3)}-1\right)+\left(13^{\operatorname{gcd}(2,9)}-1\right)\right]\right\} \\
& +\frac{1}{144}\left\{\left[\left(13^{\operatorname{gcd}(2,4)}-1\right)\right]+\left[\left(13^{\operatorname{gcd}(2,2)}-1\right)\right]\right\} \\
& +\frac{1}{144}\left\{\left[\left(13^{\operatorname{gcd}(2,5)}-1\right)+\left(13^{\operatorname{gcd}(2,11)}-1\right)\right]+\left[\left(13^{\operatorname{gcd}(2,6)}-1\right),\right]\right\}+1 .
\end{aligned}
$$

because

$$
\begin{aligned}
& 1 \frac{2}{\operatorname{gcd}(2,12)} 4^{\frac{12}{\operatorname{gcd}(2,12)}} \\
& =2^{\frac{2}{\operatorname{gcd}(2,5)}} 4^{\frac{5}{\operatorname{gcd}(2,5)}}=2^{\frac{2}{\operatorname{gcd}(2,11)}} 4^{\frac{11}{\operatorname{gcd}(2,11)}} \\
& =3^{\frac{2}{\operatorname{gcd}(2,8)}} 4^{\frac{8}{\operatorname{gcd}(2,8)}} \\
& =4^{\frac{2}{\operatorname{gcd}(2,10)}} 4^{\frac{10}{\operatorname{gcd}(2,10)}} \\
& =5^{\frac{2}{\operatorname{gcd}(2,3)}} 4^{\frac{3}{\operatorname{gcd}(2,3)}}=5^{\frac{2}{\operatorname{gcd}(2,9)}} 4^{\frac{9}{\operatorname{gcd}(2,9)}} \\
& =6^{\frac{2}{\operatorname{scd}(2,1)}} 4^{\frac{1}{\operatorname{gcd}(2,1)}}=6^{\frac{2}{\operatorname{gcd}(2,7)}} 4^{\frac{7}{\operatorname{cod}(2,7)}} \\
& =7^{\frac{2}{\operatorname{gcd}(2,1)}} 4^{\frac{1}{\operatorname{gcd}(2,1)}}=7^{\frac{2}{\operatorname{gcd}(2,7)}} 4^{\frac{7}{\operatorname{gcd}(2,7)}} \\
& =8^{\frac{2}{\operatorname{gcd}(2,3)}} 4^{\frac{3}{\operatorname{gcd}(2,3)}}=8^{\frac{2}{\operatorname{gcd}(2,9)}} 4^{\frac{9}{\operatorname{gcd}(2,9)}} \\
& =9 \frac{2}{\operatorname{scd}(2,4)} 4 \frac{4}{\operatorname{gcd}(2,4)} \\
& =10^{\frac{2}{\operatorname{gcd}(2,2)}} 4^{\frac{2}{\operatorname{gcd}(2,2)}} \\
& =11^{\frac{2}{\operatorname{gcd}(2,5)}} 4^{\frac{5}{\operatorname{gcd}(2,5)}}=11^{\frac{2}{\operatorname{gcd}(2,11)}} 4^{\frac{11}{\operatorname{gcd}(2,11)}} \\
& =12^{\frac{2}{\operatorname{gcd}(2,6)}} 4^{\frac{6}{\operatorname{gcd}(2,6)}}=1,
\end{aligned}
$$

and therefore

$$
\begin{aligned}
M_{13,4}(2)= & \frac{1}{144}\left[\left(13^{2}-1\right)+[(13-1)+(13-1)]+\left(13^{2}-1\right)+\left(13^{2}-1\right)+[(13-1)+(13-1)]\right] \\
& +\frac{1}{144}[[(13-1)+(13-1)]+[(13-1)+(13-1)]+[(13-1)+(13-1)]] \\
& +\frac{1}{144}\left[\left(13^{2}-1\right)+\left(13^{2}-1\right)+[(13-1)+(13-1)]+\left(13^{2}-1\right)\right]+1=9 .
\end{aligned}
$$

Note that setting $\lambda=1$ in (4) gives the number of defining polynomials for quasi-cyclic codes [14]

$$
M_{q, 1}(m)=\frac{1}{(q-1) m} \sum_{i \mid m} \phi(i) \operatorname{gcd}(i, q-1)\left(q^{m / i}-1\right)+1 .
$$

Table 1 gives the number of defining polynomials over $\mathbb{F}_{2}, \mathbb{F}_{3}, \mathbb{F}_{5}, \mathbb{F}_{7}$, and $\mathbb{F}_{11}$ with $\lambda=1$, Table 2 gives the number of defining polynomials over $\mathbb{F}_{4}, \mathbb{F}_{8}, \mathbb{F}_{9}$, and $\mathbb{F}_{16}$ with $\lambda=1$, and Table 3 gives the number of defining over $\mathbb{F}_{13}, \mathbb{F}_{17}$, and $\mathbb{F}_{19}$ with $\lambda=1$. To illustrate the effect of $\lambda$, Tables 4 and 5 give the number of defining polynomials over $\mathbb{F}_{3}$ and $\mathbb{F}_{4}$. 
Table 1. The number of defining polynomials over $\mathbb{F}_{2}, \mathbb{F}_{3}, \mathbb{F}_{5}, \mathbb{F}_{7}$, and $\mathbb{F}_{11}$ with $\lambda=1$

\begin{tabular}{|l|lllll|}
\hline & \multicolumn{5}{|c|}{$q$} \\
$m$ & 2 & 3 & 5 & 7 & 11 \\
\hline 1 & 2 & 2 & 2 & 2 & 2 \\
\hline 2 & 3 & 4 & 5 & 6 & 8 \\
\hline 3 & 4 & 6 & 12 & 22 & 46 \\
\hline 4 & 6 & 14 & 45 & 106 & 374 \\
\hline 5 & 8 & 26 & 158 & 562 & 3226 \\
\hline 6 & 14 & 68 & 665 & 3298 & 29576 \\
\hline 7 & 20 & 158 & 2792 & 19610 & 278390 \\
\hline 8 & 36 & 424 & 12255 & 120206 & 2679860 \\
\hline 9 & 60 & 1098 & 54262 & 747330 & 26199450 \\
\hline 10 & 108 & 2980 & 244301 & 4708486 & 259377496 \\
\hline 11 & 188 & 8054 & 1109732 & 29959498 & 2593742462 \\
\hline 12 & 352 & 22218 & 5086965 & 192243598 & 26153599626 \\
\hline
\end{tabular}

Table 2. The number of defining polynomials over $\mathbb{F}_{4}, \mathbb{F}_{8}, \mathbb{F}_{9}$, and $\mathbb{F}_{16}$ with $\lambda=1$

\begin{tabular}{|l|llll|}
\hline & \multicolumn{5}{|c|}{$q$} \\
$m$ & 4 & 8 & $9{ }^{-1}$ & 16 \\
\hline 1 & 2 & 2 & 2 & 2 \\
\hline 2 & 4 & 6 & 7 & 10 \\
\hline 3 & 10 & 26 & 32 & 94 \\
\hline 4 & 24 & 150 & 213 & 1098 \\
\hline 5 & 70 & 938 & 1478 & 13986 \\
\hline 6 & 238 & 6258 & 11107 & 186478 \\
\hline 7 & 782 & 42806 & 85412 & 2556530 \\
\hline 8 & 2744 & 299670 & 672825 & 35791946 \\
\hline 9 & 9726 & 2130458 & 5380862 & 509033346 \\
\hline 10 & 34990 & 15339642 & 43586287 & 7330084546 \\
\hline 11 & 127102 & 111557594 & 356602952 & 106619309362 \\
\hline 12 & 466198 & 818092242 & 2941985613 & 1563749966062 \\
\hline
\end{tabular}

\section{Quasi-twisted codes over $\mathbb{F}_{17}$ and $\mathbb{F}_{19}$}

In this section, the defining polynomials given above are used to construct quasi-twisted codes over $\mathbb{F}_{17}$ and $\mathbb{F}_{19}$. The number of defining polynomials over $\mathbb{F}_{17}$ for $m=1$ to 5 is given in Table 6 and over $\mathbb{F}_{19}$ for $m=1$ to 5 in Table 7 . Note that the zero polynomial is not considered in constructing codes.

Considering a code structure (i.e. QT), results in a search space that is smaller than for the general code design problem. The more restrictions on the structure, the smaller the search, but this creates a tradeoff since good codes may be missed if too much structure is imposed on the code. The QT codes presented here were constructed using a stochastic optimization algorithm, namely tabu search, which is similar to that in $[10,11,14]$. By restricting the search for good codes to the class of QT codes, and using a stochastic heuristic, codes with high minimum distance can be found with a reasonable amount of computational effort. Based on the results obtained here, this approach provides a good tradeoff.

The search for a $(p m, m)$ QT code begins with a random set of $p$ defining polynomials. A polynomial 
Table 3. The number of defining polynomials over $\mathbb{F}_{13}, \mathbb{F}_{17}$, and $\mathbb{F}_{19}$ with $\lambda=1$

\begin{tabular}{|l|lll|}
\hline & \multicolumn{3}{|l|}{$q$} \\
$m$ & 13 & 17 & 19 \\
\hline 1 & 2 & 2 & 2 \\
\hline 2 & 9 & 11 & 12 \\
\hline 3 & 64 & 104 & 130 \\
\hline 4 & 605 & 1317 & 1822 \\
\hline 5 & 6190 & 17750 & 27514 \\
\hline 6 & 67117 & 251543 & 435760 \\
\hline 7 & 747008 & 3663740 & 7094222 \\
\hline 8 & 8497807 & 54499433 & 117943232 \\
\hline 9 & 98189934 & 823526990 & 1991899630 \\
\hline 10 & 1148826961 & 12599979635 & 34061506732 \\
\hline 11 & 13576972684 & 194726683568 & 588334640902 \\
\hline 12 & 161792326165 & 3034491071421 & 10246828768390 \\
\hline
\end{tabular}

Table 4. The number of defining polynomials over $\mathbb{F}_{3}$ with $\lambda \in\{1,2\}$

\begin{tabular}{|l|l|l|}
\hline$m$ & $\lambda$ & Number \\
\hline 1 & 1,2 & 2 \\
\hline 2 & 1 & 4 \\
\hline 2 & 2 & 3 \\
\hline 3 & 1,2 & 6 \\
\hline 4 & 1 & 14 \\
\hline 4 & 2 & 11 \\
\hline 5 & 1,2 & 26 \\
\hline 6 & 1 & 68 \\
\hline 6 & 2 & 63 \\
\hline 7 & 1,2 & 158 \\
\hline 8 & 1 & 424 \\
\hline 8 & 2 & 411 \\
\hline 9 & 1,2 & 1098 \\
\hline 10 & 1 & 2980 \\
\hline 10 & 2 & 2955 \\
\hline 11 & 1,2 & 8054 \\
\hline 12 & 1 & 22218 \\
\hline 12 & 2 & 22151 \\
\hline
\end{tabular}

is replaced with a new polynomial if this results in an increase in the minimum distance. This process is repeated until a code with the desired minimum distance is found or an iteration threshold is reached. The search is restarted periodically to ensure good coverage of the search space. It is not necessary to check the weight of every codeword in a QT code in order to determine the minimum distance $d$. Only a subset of the codewords need be considered since the Hamming weights of the polynomials $r_{j}(x)$ and $r_{i}(x)$ are the same if

$$
r_{j}(x)=\gamma x^{l} r_{i}(x) \bmod \left(x^{m}-\lambda\right),
$$

for integer $l \geq 0$ and scalar $\gamma \in \mathbb{F}_{q} \backslash\{0\}$. 
Table 5. The number of defining polynomials over $\mathbb{F}_{4}$ with $\lambda \in\left\{1, \alpha, \alpha^{2}\right\}$

\begin{tabular}{|l|l|l|}
\hline$m$ & $\lambda$ & Number \\
\hline 1 & $1, \alpha, \alpha^{2}$ & 2 \\
\hline 2 & $1, \alpha, \alpha^{2}$ & 4 \\
\hline 3 & 1 & 10 \\
\hline 3 & $\alpha, \alpha^{2}$ & 8 \\
\hline 4 & $1, \alpha, \alpha^{2}$ & 24 \\
\hline 5 & $1, \alpha, \alpha^{2}$ & 70 \\
\hline 6 & 1 & 238 \\
\hline 6 & $\alpha, \alpha^{2}$ & 232 \\
\hline 7 & $1, \alpha, \alpha^{2}$ & 782 \\
\hline 8 & $1, \alpha, \alpha^{2}$ & 2744 \\
\hline 9 & 1 & 9726 \\
\hline 9 & $\alpha, \alpha^{2}$ & 9710 \\
\hline 10 & $1, \alpha, \alpha^{2}$ & 34990 \\
\hline 11 & $1, \alpha, \alpha^{2}$ & 127102 \\
\hline 12 & 1 & 466198 \\
\hline 12 & $\alpha, \alpha^{2}$ & 466152 \\
\hline
\end{tabular}

Table 6. The number of defining polynomials over $\mathbb{F}_{17}$

\begin{tabular}{|l|l|l|}
\hline$m$ & $\lambda$ & Number \\
\hline 1 & all & 2 \\
2 & $1,2,4,8,9,13,15,16$ & 11 \\
2 & $3,5,6,7,10,11,12,14$ & 10 \\
3 & all & 104 \\
4 & $1,4,13,16$ & 1317 \\
4 & $2,8,9,15$ & 1315 \\
4 & $3,5,6,7,10,11,12,14$ & 1306 \\
5 & all & 17750 \\
6 & $1,2,4,8,9,13,15,16$ & 251543 \\
6 & $3,5,6,7,10,11,12,14$ & 251440 \\
\hline
\end{tabular}

The best QT codes found over $\mathbb{F}_{17}$ are given in Tables 8 to 10 , and over $\mathbb{F}_{19}$ in Tables 11 to 13. The defining polynomials are listed with the lowest degree coefficient on the left, i.e. 7321 corresponds to the polynomial $x^{3}+2 x^{2}+3 x+7$, with leading zeroes left out for brevity. The digits $10,11, \ldots, 18$ are denoted by $(10),(11), \ldots,(18)$, respectively. As an example, consider the [24,4] code in Table 12 with $m=4, \lambda=1$ and $p=6$ defining polynomials. These polynomials give the following generator matrix

$$
G=\left[\begin{array}{l|l|l|l|l|l}
1129 & 1596 & 1632 & 01(14)(11) & 0016 & 169(12) \\
9112 & 6159 & 2163 & (11) 01(14) & 6001 & (12) 169 \\
2911 & 9615 & 3216 & (14)(11) 01 & 1600 & 9(12) 16 \\
1291 & 5961 & 6321 & 1(14)(11) 0 & 0160 & 69(12) 1
\end{array}\right]
$$

with weight distribution 
Table 7. The number of defining polynomials over $\mathbb{F}_{19}$

\begin{tabular}{|l|l|l|}
\hline$m$ & $\lambda$ & number \\
\hline 1 & all & 2 \\
2 & $1,4,5,6,7,9,11,16,17$ & 12 \\
2 & $2,3,8,10,12,13,14,15,18$ & 11 \\
3 & $1,7,8,11,12,18$ & 130 \\
3 & $2,3,4,5,6,9,10,13,14,15,16,17$ & 128 \\
4 & $1,4,5,6,7,9,11,16,17$ & 1822 \\
4 & $2,3,8,10,12,13,14,15,18$ & 1811 \\
5 & all & 27514 \\
\hline
\end{tabular}

$\begin{array}{cr}i & W_{i} \\ 0 & 1 \\ 20 & 6372 \\ 21 & 10944 \\ 22 & 28296 \\ 23 & 49680 \\ 24 & 35028\end{array}$

This code is optimal since it meets the Griesmer bound (1), and so establishes that $d_{19}(24,4)=20$. The codes that meet the Griesmer bound are indicated by ${ }^{*}$ in the tables. The codes given in the tables are QC codes $(\lambda=1)$ when a QC code has the highest minimum distance among all QT codes with the same length and dimension. In two cases for $q=19$ and $m=4$, a code with $\lambda=-1$ was found with a minimum distance higher than the corresponding QC code.

\section{Conclusion}

Closed-form expressions for the number of twistulant matrices and corresponding defining polynomials were given. These polynomials were used in the construction of quasi-twisted codes, and several new optimal codes were obtained.

Acknowledgment: The authors would like to thank Jumah Ali Algallaf for computing the numbers of defining polynomials.

\section{References}

[1] K. Betsumiya, S. Georgiou, T. A. Gulliver, M. Harada, C. Koukouvinos, On self-dual codes over some prime fields, Disc. Math. 262(1-3) (2003) 37-58.

[2] W. Bosma, J. Cannon, C. Playoust, The Magma algebra system I: The user language, J. Symbolic Comput., 24(3-4) (1997) 235-265.

[3] E. Z. Chen, N. Aydin, New quasi-twisted codes over $F_{11}-$ minimum distance bounds and a new database, J. Inform. Optimization Sci., 36(1-2) (2015) 129-157. 
[4] E. Z. Chen, N. Aydin, A database of linear codes over $\mathbb{F}_{13}$ with minimum distance bounds and new quasi-twisted codes from a heuristic search algorithm, J. Algebra Comb. Discrete Appl., 2(1) (2015) $1-16$.

[5] J. A. Gallian, Contemporary Abstract Algebra, Eighth Edition, Brooks/Cole, Boston, MA 2013.

[6] M. Grassl, Code Tables: Bounds on the parameters of various types of codes, available online at http://www . codetables.de.

[7] P.P. Greenough, R. Hill, Optimal ternary quasi-cyclic codes, Des. Codes, Cryptogr. 2(1) (1992) 81-91.

[8] T. A. Gulliver, Quasi-twisted codes over $F_{11}$, Ars Combinatoria 99 (2011) 3-17.

[9] T. A. Gulliver, New optimal ternary linear codes, IEEE Trans. Inform. Theory 41(4) (1995), 1182-1185.

[10] T. A. Gulliver, V. K. Bhargava, Some best rate $1 / p$ and rate $(p-1) / p$ systematic quasi-cyclic codes over $G F(3)$ and $G F(4)$, IEEE Trans. Inform. Theory 38(4) (1992) 1369-1374.

[11] T. A. Gulliver, V. K. Bhargava, New good rate $(m-1) / p m$ ternary and quaternary quasi-cyclic codes, Des. Codes, Cryptogr. 7(3) (1996) 223-233.

[12] F. J. MacWilliams, N. J. A. Sloane, The Theory of Error-Correcting Codes, North-Holland, New York, NY 1977.

[13] D. W. Newhart, On minimum weight codewords in QR codes, J. Combin. Theory Ser. A 48(1) (1988) 104-119.

[14] V. Ch. Venkaiah, T. A. Gulliver, Quasi-cyclic codes over $\mathbb{F}_{13}$ and enumeration of defining polynomials, J. Discrete Algorithms 16 (2012) 249-257. 


\section{Appendix A:}

Table 8. QT codes over $\mathbb{F}_{17}$ with $m=3$

\begin{tabular}{|c|c|c|c|}
\hline code & $\lambda$ & $d$ & $b_{i}(x)$ \\
\hline$[6,3]$ & 1 & $4^{*}$ & $11,1(13) 8$ \\
\hline$[9,3]$ & 1 & $7^{*}$ & $18(11), 1,117$ \\
\hline$[12,3]$ & 1 & $10^{*}$ & $14(15), 1(12), 16,157$ \\
\hline$[15,3]$ & 1 & $13^{*}$ & $1,118,125,152,164$ \\
\hline$[18,3]$ & 1 & $16^{*}$ & $118,125,1,152,13(12), 164$ \\
\hline$[21,3]$ & 1 & $18^{*}$ & $152,14(10), 13(16), 17(12), 127,172,115$ \\
\hline$[24,3]$ & 1 & $21^{*}$ & $11,137,11(10), 145,17(16), 18(11), 15(16), 13$ \\
\hline$[27,3]$ & 1 & $24^{*}$ & $116,12(11), 12(16), 18(11), 118,11(13), 12,147,16(10)$ \\
\hline$[30,3]$ & 1 & 26 & $147,11(14), 12(10), 16,11,164,164,1(13) 8,11(12), 182$ \\
\hline$[33,3]$ & 1 & 29 & $184,17(12), 14,114,17(10), 11(10), 19(16), 18,12(13), 11(12), 1(13) 4$ \\
\hline$[36,3]$ & 1 & 32 & $18,1(13) 4,12,1(14), 1(12) 8,11(13), 14(13), 157,18(12), 198,1(10), 16(11)$ \\
\hline$[39,3]$ & 1 & $35^{*}$ & $116,124,117,1(13) 8,11(11), 11(10), 12,145,19,12(14), 1(12), 1(10) 4,15(14)$ \\
\hline$[42,3]$ & 1 & $38^{*}$ & $1(13), 15(12), 1,152,11,125,1(13) 4,12(11), 116,175,118,198,132,137$ \\
\hline$[45,3]$ & 1 & 40 & $1,1(15), 118,113,1(12) 8,14(11), 124,114,19(16), 17,126,125,154,14,11(16)$ \\
\hline$[48,3]$ & 1 & 43 & $\begin{array}{l}15,126,14(15), 1(10) 4,15(16), 17(16), 11(14), 125,12(12), 13(14), 143,11(10) \\
1(10)(11), 11(16), 16(15), 157\end{array}$ \\
\hline$[51,3]$ & 1 & 46 & $\begin{array}{l}18,126,198,11(13), 157,13(14), 15(14), 13(15), 12(16), 12(14), 12,152,13(10), \\
11,16(11), 16(14), 14(11)\end{array}$ \\
\hline$[54,3]$ & 1 & 49 & $\begin{array}{l}11,11(13), 154,1,135,12,152,124,143,137,14(11), 116,16(14), 16(15), 13(12) \\
11(12), 1(10) 8,1(10)\end{array}$ \\
\hline$[57,3]$ & 1 & $52^{*}$ & $\begin{array}{l}11,13(16), 1(13) 4,11(15), 1(13) 8,12,15(14), 16(16), 12(11), 11(12), 18(12), 14(14), 1, \\
15(16), 125,115,147,145,162\end{array}$ \\
\hline$[60,3]$ & 1 & 54 & $\begin{array}{l}12,1,113,152,12(10), 11(14), 114,16(11), 15(12), 135,1(10)(11), 17,16,126,11,134, \\
16(14), 157,125,165\end{array}$ \\
\hline$[63,3]$ & 1 & 57 & $\begin{array}{l}11(13), 11,12(15), 154,19(16), 12,12(10), 114,125,12(12), 15(14), 15(12), 117,113 \\
14(10), 11(15), 16(15), 162,1(13), 13(16), 123\end{array}$ \\
\hline$[66,3]$ & 1 & 60 & $\begin{array}{l}13,145,15(12), 13(13), 117,1(15), 12(10), 13(10), 143,116,14(13), 168,16(11), 11 \\
11(12), 13(15), 1(14), 13(14), 115,182,17,142\end{array}$ \\
\hline$[69,3]$ & 1 & 63 & $\begin{array}{l}11(13), 1(12), 154,1,19,128,12(10), 182,12(16), 12(15), 15(14), 18(12), 112,11(12), \\
117,198,15(11), 1(11), 13(16), 11(14), 138,1(13), 145\end{array}$ \\
\hline$[72,3]$ & 1 & 65 & $\begin{array}{l}15,175,11,13(12), 1(10), 11(12), 118,19(14), 17(12), 16(11), 182,154,137,138,132, \\
16(15), 145,18,11(15), 125,142,147,116,17(10)\end{array}$ \\
\hline$[75,3]$ & 1 & 68 & $\begin{array}{l}\text { 1(10), 12, 12(13), 13(15), 126, 1(11), 12(16), 112, 11(13), 117, 12(12), 16(14), 14(13), } \\
175,1(10) 4,11,14(11), 168,11(16), 17(10), 17(16), 1(12), 1(12) 8,114,127\end{array}$ \\
\hline
\end{tabular}


Table 9. QT codes over $\mathbb{F}_{17}$ with $m=4$

\begin{tabular}{|c|c|c|c|}
\hline code & $\lambda$ & $d$ & $b_{i}(x)$ \\
\hline$[8,4]$ & 1 & $5^{*}$ & 1,1128 \\
\hline$[12,4]$ & 1 & $9^{*}$ & $16(11)(14), 123,1685$ \\
\hline$[16,4]$ & 1 & $13^{*}$ & $12(10)(16), 1275,113,11(16) 7$ \\
\hline$[20,4]$ & 1 & 16 & $18,13(11) 7,113(12), 1179,12(15) 2$ \\
\hline$[24,4]$ & 1 & $20^{*}$ & $134,1798,18(11), 1(11) 1(14), 126(10), 1(10)(11)(12)$ \\
\hline$[28,4]$ & 1 & 23 & $1(11), 1(13) 1,11(10) 6,137(15), 1(15)(11), 1(11) 1(16), 1(10) 5$ \\
\hline$[32,4]$ & 1 & 27 & $1,124,13(14) 7,1(10)(11)(12), 126(16), 1(15)(16), 1437,13(13) 2$ \\
\hline$[36,4]$ & 1 & 30 & $13,15(16)(11), 13(15)(14), 1(10) 2,16(16), 11,112(14), 12(13)(16), 1734$ \\
\hline$[40,4]$ & 1 & 34 & $14(10) 7,1453,1317,1,1(13) 1,11(15) 4,1598,12(15) 4,1(12) 2,118(10)$ \\
\hline$[44,4]$ & 1 & 38 & $\begin{array}{l}114(15), 12(10)(16), 11,118(14), 113,17(13)(16), 1(16) 7,14(15)(10), 1(12) 7,19(14), \\
147(16)\end{array}$ \\
\hline$[48,4]$ & 1 & 41 & $\begin{array}{l}12(12), 12,11(16)(14), 181,14,111(14), 1254,12(10)(13), 1114,1(10) 5,156(10) \\
147(12)\end{array}$ \\
\hline$[52,4]$ & 1 & 45 & $\begin{array}{l}124(16), 14(13) 3,1114,16,1(15)(16), 12,183(10), 118(16), 1192,13(10), 12(14), \\
173(11), 14(15)(10) \\
116(15), 14(10) 2,1(11) 64,14(14) 7\end{array}$ \\
\hline$[60,4]$ & 1 & 52 & $\begin{array}{l}11,11(13), 13(16), 146(13), 153,13,192,164(10), 1386,137(13), 1372,125(16) \\
11(10) 5,1272,12(13) 3\end{array}$ \\
\hline$[64,4]$ & 1 & 56 & $\begin{array}{l}13,158(14), 11(15)(14), 1982,1(13) 5,1(10) 1(16), 12(14) 5,1517,13(13)(12), 103 \\
11(12) 9,1(12)(15) 8,116(16), 11(15) 7,1239,1(12)(10)\end{array}$ \\
\hline$[68,4]$ & 1 & 59 & $\begin{array}{l}12,1(10) 1(12), 12(13)(12), 1(11) 2,151(11), 17(12)(15), 16(11)(12), 1(15)(16), 1187 \\
1472,124(15), 13(10)(13), 11(14), 1462,1(11) 3,172(14), 11\end{array}$ \\
\hline$[72,4]$ & 1 & 63 & $\begin{array}{l}1(15), 12(10) 2,11(16)(11), 11(16) 7,1(13), 15(16) 5,1838,12(15), 14(15), 13(13)(11) \\
1682,1(12) 38,1468,135(15), 1795,137(10), 14(10), 175\end{array}$ \\
\hline$[76,4]$ & 1 & 67 & $\begin{array}{l}1(10), 12(16) 7,15(12) 8,1127,13(15) 5,12(15) 6,1358,126(16), 1123,121(13), 14,1387, \\
187,1654,11(12)(14), 1193,163(11), 1(12)(13), 1468\end{array}$ \\
\hline$[80,4]$ & 1 & 70 & $\begin{array}{l}11(13), 1,13,1(10) 1(11), 15(12) 4,1268,167,11(12), 141(10), 184(15), 1517,17(12)(11) \\
16(15)(14), 12(14)(15), 18(14), 1188,17(10)(14), 138(12), 111(16), 176(16)\end{array}$ \\
\hline$[84,4]$ & 1 & 74 & $\begin{array}{l}1418,17,1356,135(15), 13(16) 2,12(11)(13), 136,1162,16(10)(15), 149(16), 147(13) \\
12(16)(13), 17(16), 11(14) 5,1164,1213,121(15), 1838,11(11) 5,1547,17(10) 4\end{array}$ \\
\hline$[88,4]$ & 1 & 78 & $\begin{array}{l}1(10), 12(11)(16), 1(11)(13), 15(14), 13(16) 8,138(11), 133,172(15), 1568,13(16)(10), \\
132(14), 1(13)(15), 1169,1(11) 1,17(15), 1146,14(13) 2,14(10)(11), 11(10)(11), \\
14(11)(15), 14(13)(14), 15(11)(12)\end{array}$ \\
\hline$[92,4]$ & 1 & 81 & $\begin{array}{l}13,11(16) 2,17(12) 5,16(15)(12), 11(10)(11), 1(13)(16), 1(10)(11), 119(11), 16(14) \\
163(11), 139,1(12) 2,13(13) 8,15(16), 162,128(13), 1652,14(12), 16(16) 5,162(14) \\
118(15), 11(10) 7,1(10) 1\end{array}$ \\
\hline$[96,4]$ & 1 & 85 & $\begin{array}{l}1,16(11)(14), 1145,16(10)(14), 102,14(11)(12), 1(10) 8(11), 111,11(11) 2,13(13) 8 \\
176(10), 16(11) 8,1376,12(11) 5,1(10) 8(12), 187,15(12) 3,1(12) 3(11), 11(12) 4 \\
17(12)(10), 111(12), 1(10)(12), 125(14), 1832\end{array}$ \\
\hline$[100,4]$ & 1 & 89 & $\begin{array}{l}105,1(12) 3,1235,198(12), 13(15) 3,151(15), 134(10), 11,146(10), 1(13)(15), 1(11) 4, \\
11(12)(11), 184(15), 143(15), 113(14), 1158,17(12)(10), 1173,116(10), 183(10), \\
119(11), 19(15) 4,11(15)(13), 1584,1425\end{array}$ \\
\hline
\end{tabular}


Table 10. QT codes over $\mathbb{F}_{17}$ with $m=5$

\begin{tabular}{|c|c|c|c|}
\hline code & $\lambda$ & $d$ & $b_{i}(x)$ \\
\hline$[10,5]$ & 1 & $6^{*}$ & $18,16(15)(16) 2$ \\
\hline$[15,5]$ & 1 & 10 & $11,162(15)(14), 11(11)(15)(12)$ \\
\hline$[20,5]$ & 1 & 14 & $131,11(13) 96,1041,11(11)$ \\
\hline$[25,5]$ & 1 & 19 & $15,11(11)(16)(12), 135(12) 4,1126(10), 1278(14)$ \\
\hline$[30,5]$ & 1 & 23 & $11(13)(10)(15), 1(11)(10)(16), 115,115(11) 4,112(11) 8,17(12) 8(11)$ \\
\hline$[35,5]$ & 1 & 28 & $111(16) 6,143(13)(16), 121,106(13), 15(14) 68,119(16) 6,13(13)(16) 7$ \\
\hline$[40,5]$ & 1 & 32 & $19,13415,1576(16), 1547,17(13)(15)(14), 12(12)(13)(12), 13(12) 85,12(13)(11) 4$ \\
\hline$[45,5]$ & 1 & 36 & $\begin{array}{l}1(16) 58,131,13(12) 9,1(15) 7(16), 11(13)(15)(14), 12(15) 3(15), 1(10) 1(10) \\
13(14) 3(12), 141(15)(12)\end{array}$ \\
\hline$[50,5]$ & 1 & 41 & $\begin{array}{l}\text { 16, 121(15)(13), 15217, 172(13)(12), 11132, 17(10)1(16), 13(11)75, 14(14)2(15), } \\
12(11) 26,11(15) 7(14)\end{array}$ \\
\hline$[55,5]$ & 1 & 45 & $\begin{array}{l}1,12,11(16)(14)(15), 1(15)(11) 4,1416(10), 11636,19(10) 7,111(15) 8,11(13) 7(16) \\
12495,1748\end{array}$ \\
\hline$[60,5]$ & 1 & 50 & $\begin{array}{l}\text { 19, 10(11)4, 1(10)83(11), 1(14)74, 11(13)(16)3, 11452, 13(10)(15)(14), 13(12)(14)(16), } \\
12(12) 34,12(13) 8(15), 17(10), 154(15) 3\end{array}$ \\
\hline$[65,5]$ & 1 & 54 & $\begin{array}{l}13,11,1058,14(14) 4,126(14) 8,13(11)(12)(10), 121(11) 8,1389(12), 19(15) 3,129(12), \\
13(14) 94,12(15) 27,1212(13)\end{array}$ \\
\hline$[70,5]$ & 1 & 59 & $\begin{array}{l}11,13475,1(12)(10)(14), 11(13) 4,18(12)(15) 2,14(12) 4,14(13)(16) 3,173(11)(12) \\
11235,1586(11), 1(16) 6(12), 18(14)(10), 15(12) 1(12), 1(14) 9(10)\end{array}$ \\
\hline$[75,5]$ & 1 & 63 & $\begin{array}{l}1(10)(11), 1956,19,16(15)(12)(16), 19(16) 4,11(14) 59,112(10), 15(11)(12) 8 \\
17(14) 3,17(16) 42,11(10) 4(15), 11(11) 3,17(16) 1(14), 12(15) 1,19(14) 8\end{array}$ \\
\hline$[80,5]$ & 1 & 68 & $\begin{array}{l}1,1(16)(14) 6,14,13(14) 6(15), 163(11)(12), 111,17595,179(15)(14), 14382, \\
1(12) 5(12)(10), 1(13)(14) 9,1(12)(15) 6(16), 14(10) 2,12(14) 15,19(13) 82,1825(11)\end{array}$ \\
\hline$[85,5]$ & 1 & 72 & $\begin{array}{l}1(10), 1327(16), 11,1(11) 11,177,15(10) 4,11513,13263,155(14), 12(16) 8(14) \\
1342(11), 123(15) 9,125(13)(14), 12(14) 38,1(12) 5(12), 17(10) 4(10), 1641(14)\end{array}$ \\
\hline$[90,5]$ & 1 & 77 & $\begin{array}{l}1(11), 119(13), 1933,18198,13714,115(15)(15), 195(13), 17(11) 3,11475,13(15) 7 \\
156(10) 2,1(11)(16)(10), 15(16) 2(15), 15(11) 34,113,11(10)(12) 3,1(10) 5(13) \\
11(14)(10) 3\end{array}$ \\
\hline$[95,5]$ & 1 & 81 & $\begin{array}{l}1,12432,1438(10), 13(11) 1(12), 1(11) 42,131(10)(11), 14(11) 1(15), 1425(11), 10(10), \\
1185(13), 1(12) 18,15982,118(15) 3,1794(14), 12927,1321(14), 13(14)(16)(14), \\
12(14)(12)(15), 1976(16)\end{array}$ \\
\hline$[100,5]$ & 1 & 86 & $\begin{array}{l}16(14)(13)(11), 15(14) 72,11,1,1431(11), 12(10)(13) 3,113(12)(14), 1156(10) \\
115(11), 1752(16), 12(12)(10)(13), 1247,173(13) 4,172(15)(16), 1(13)(11)(11) \\
116(11)(14), 146(12), 16(15)(16) 8,11(11)(15) 3,157(16) 4\end{array}$ \\
\hline$[105,5]$ & 1 & 90 & $\begin{array}{l}13(13)(10) 5,1(11) 37,11,1(14)(11) 3,117(14) 3,13(11)(16)(12), 13,123(16) 7,1195 \\
19(12) 9,15(16)(12), 11(14) 39,11(14) 6(14), 1(12) 8,15932,1517(10), 1(12)(11) 9 \\
156(10)(12), 13(10) 6(13), 1183(13), 11(10)(16)\end{array}$ \\
\hline$[110,5]$ & 1 & 95 & $\begin{array}{l}12,14,13(12) 9,1(15) 7(16), 11(13)(15)(14), 12(15) 3(15), 1(10) 1(10), 13(14) 3(12), \\
141(15)(12), 15168,11534,1568(14), 118(15)(12), 1(10) 44,135(11) 3,163(11)(12), \\
11(15)(10)(16), 13(11)(15)(14), 169(16)(10), 117(11), 12(11)(14)(10), 14234\end{array}$ \\
\hline$[115,5]$ & 1 & 99 & $\begin{array}{l}18,1,11(10) 3(16), 1(11)(12)(15) 8,13(16) 9(11), 13(16) 95,13(16) 54,1012,1127(14) \\
15942,131(16) 2,1187,12(12) 9(12), 1326,15(14) 1(14), 11112,18(11)(12) 8,15(11) 32, \\
164(10) 8,159(12) 5,1(14)(16)(13), 103(13), 17(11) 4\end{array}$ \\
\hline$[120,5]$ & 1 & 104 & $\begin{array}{l}14,117(15) 8,1071,15(13)(11)(15), 15(12) 1,13(12)(16)(13), 14(15) 62,134(10)(13) \\
15(16) 85,15(14)(16), 12,19(11)(12), 1815,112(14)(13), 14(11) 2,15(14)(10) 8 \\
12(12)(11)(15), 1256(13), 1(12) 3(12), 1452(16), 1243(10), 1166(14), 13(11)(10) 4 \\
11(10) 5(15)\end{array}$ \\
\hline$[125,5]$ & 1 & 109 & $\begin{array}{l}1(10), 13184,13(13)(16), 12(13) 53,1081,1(11)(10)(10), 13(11) 2,15(14)(15) 2, \\
11(14)(11) 9,127(16)(13), 1415(12), 12972,15175,11(11)(12) 6,1(13)(16)(13), 19832, \\
14295,13(16)(11), 12(16) 4(15), 132(14)(15), 156(14) 2,11(12) 87,131(11) 4,15(16) 64, \\
1656\end{array}$ \\
\hline
\end{tabular}


Table 11. QT codes over $\mathbb{F}_{19}$ with $m=3$

\begin{tabular}{|c|c|c|c|}
\hline code & $\lambda$ & $d$ & $b_{i}(x)$ \\
\hline$[6,3]$ & 1 & $4^{*}$ & 1,112 \\
\hline$[9,3]$ & 1 & $7^{*}$ & $135,11,16(16)$ \\
\hline$[12,3]$ & 1 & $10^{*}$ & $1,15(12), 17(15), 138$ \\
\hline$[15,3]$ & 1 & $13^{*}$ & $1(11), 154,11(16), 149,14(17$ \\
\hline$[18,3]$ & 1 & $16^{*}$ & $17,159,11(16), 157,145,176$ \\
\hline$[21,3]$ & 1 & 18 & $1,114,142,12,137,159,16(15)$ \\
\hline$[24,3]$ & 1 & $21^{*}$ & $11,15(12), 1(11)(18), 138,15(17), 139,1(10) 6,159$ \\
\hline$[27,3]$ & 1 & $24^{*}$ & $12,18(14), 116,162,189,154,14(14), 13(12), 11(14)$ \\
\hline$[30,3]$ & 1 & $27^{*}$ & $1(11), 1,15(11), 1(10) 7,1(13) 6,135,11(13), 1(10)(18), 15(16), 128$ \\
\hline$[33,3]$ & 1 & 29 & $112,115,1(12)(14), 12,11,15(12), 138,1(17), 1(16), 128,192$ \\
\hline$[36,3]$ & 1 & 32 & $14(15), 15,125,117,12(13), 158,18(17), 11(13), 13(12), 1,17(15), 11(17)$ \\
\hline$[39,3]$ & 1 & 35 & $167,16,11(11), 1(14), 17(12), 14,1(12)(14), 173,17,13(16), 146,127,152$ \\
\hline$[42,3]$ & 1 & $38^{*}$ & $\begin{array}{l}\begin{array}{l}1(11), 11(17), 17(18), 114,12,12(12), 15(17), 1(13) 6,18(17), 162,11(12), 19,116 \\
1(10)(18)\end{array}\end{array}$ \\
\hline$[45,3]$ & 1 & $41^{*}$ & $\begin{array}{l}\begin{array}{l}14,1(14) 6,16(14), 17,11(11), 162,17(12), 187,16,1(16), 1(10)(15), 1(10) 6,11(12) \\
178,169\end{array}\end{array}$ \\
\hline$[48,3]$ & 1 & 43 & $\begin{array}{l}15(16), 1,168,12(12), 19(14), 129,1(16), 12,1(12) 9,154,135,189,112,1(10) 9 \\
1(10) 8,117\end{array}$ \\
\hline$[51,3]$ & 1 & 46 & $\begin{array}{l}12,18(17), 162,15(11), 11(11), 137,13(14), 15,17(17), 11,163,1,11(13), 12(15) \\
19(14), 135,18(15)\end{array}$ \\
\hline$[54,3]$ & 1 & 49 & $\begin{array}{l}1,18,176,13(16), 162,12(12), 1(10), 125,154,192,147,1(10) 6,135,159,134,169 \\
13(12), 17\end{array}$ \\
\hline$[57,3]$ & 1 & 52 & $\begin{array}{l}14,13,1,1(13) 6,129,143,1(10), 168,118,158,124,1(10)(18), 18(14), 19(14), 14(15) \\
1(11)(18), 159,12(13), 14(14)\end{array}$ \\
\hline$[60,3]$ & 1 & 55 & $\begin{array}{l}1(14), 1(10), 14,1(17), 115,126,17(18), 154,17,1(13) 6,129,12(15), 137,11(16), 128 \\
1(11)(18), 1(13)(18), 127,113,149\end{array}$ \\
\hline$[63,3]$ & 1 & 57 & $\begin{array}{l}17,1(12) 9,1(16), 1(14), 193,135,1(10) 6,12(16), 19,1(18), 162,116,145,126,129 \\
169,14(17), 15(12), 11(16), 1(12), 1(14) 6\end{array}$ \\
\hline$[66,3]$ & 1 & 60 & $\begin{array}{l}1(12), 11(15), 15(18), 176,137,193,138,12(11), 1(10) 9,1(11)(18), 13(16), 135,162 \\
11(13), 16(15), 1(16) 6,17(18), 132,17,1(12)(14), 147,125\end{array}$ \\
\hline$[69,3]$ & 1 & 63 & $\begin{array}{l}1,18,1(16), 134,1(13)(18), 14(15), 114,1(14), 115,11(11), 139,186,15(16), 12(15) \\
1(13), 15(17), 129,1(10) 8,17(18), 14(11), 132,14(14), 126\end{array}$ \\
\hline$[72,3]$ & 1 & 66 & $\begin{array}{l}1(18), 12(15), 173,117,139,1(15), 15(18), 1(12) 9,167,1(13), 11,127,1(13) 6,11(16) \\
11(14), 13(16), 19,168,129,13(17), 1(11)(18), 138,14(11), 16(16)\end{array}$ \\
\hline$[75,3]$ & 1 & 69 & $\begin{array}{l}15,12(14), 13(17), 149,1,1(14), 193,11(13), 134,16(16), 1(13)(18), 15(16), 115,1(13) \\
14(15), 12(17), 1(11)(18), 128,116,16(14), 17(17), 15(12), 186,137,11\end{array}$ \\
\hline
\end{tabular}


Table 12. QT codes over $\mathbb{F}_{19}$ with $m=4$

\begin{tabular}{|c|c|c|c|}
\hline code & $\lambda$ & $d$ & $b_{i}(x)$ \\
\hline$[8,4]$ & 1 & $5^{*}$ & 12,114 \\
\hline$[12,4]$ & 1 & $9^{*}$ & $1(10), 1193,1(10) 6(15)$ \\
\hline$[16,4]$ & -1 & $13^{*}$ & $12(12), 16,134(12), 111(14)$ \\
\hline$[20,4]$ & -1 & $17^{*}$ & $14(17), 115(17), 1281,11(11) 9,1146$ \\
\hline$[24,4]$ & 1 & $20^{*}$ & $1129,1596,1632,1(14)(11), 16,169(12)$ \\
\hline$[28,4]$ & 1 & 23 & $18,135(14), 19,16(14)(17), 11(15), 145(11), 14(11)(15)$ \\
\hline$[32,4]$ & 1 & 27 & $104,112,1534,1629,1737,17(15) 6,12(16) 6,1356$ \\
\hline$[36,4]$ & 1 & 30 & $18,119(12), 12(16), 112,15(11)(15), 13(16) 3,121(18), 15(18)(11), 1719$ \\
\hline$[40,4]$ & 1 & 34 & $\begin{array}{l}106,14(14) 3,1627,11(12)(18), 16(10)(14), 14(11)(12), 157(15), 152(16), 1(16) 9 \\
1923\end{array}$ \\
\hline$[44,4]$ & 1 & 38 & $16,127(14), 12(14)(16), 15,1(10)(18) 7,18(17) 6,149(15), 1398,158,1279,141(18)$ \\
\hline$[48,4]$ & 1 & 41 & $\begin{array}{l}\begin{array}{l}159(14), 11,15(17), 121(13), 15(13)(16), 14(10)(17), 12(14) 6,124(18), 146(11), 13(11) \\
1168,118(15)\end{array}\end{array}$ \\
\hline$[52,4]$ & 1 & 45 & $\begin{array}{l}\text { 1(12), 1(12)5, 14(11), 123(18), 14(10)(18), 13(15)(12), 12(14)(11), 15, 187(17) } \\
163(17), 159(11), 17(12), 1174\end{array}$ \\
\hline$[56,4]$ & 1 & 49 & $\begin{array}{l}13,11(15)(13), 1(10) 5(14), 1(11) 59,161(14), 1619,13(10)(15), 1(12)(16), 11(12)(11) \\
1(10)(12), 12(15)(18), 13(16) 6,126(11), 1272\end{array}$ \\
\hline$[60,4]$ & 1 & 52 & $\begin{array}{l}1843,169,17(17), 1153,11(11) 2,1(11) 8(14), 183(17), 16(16) 2,141(12), 12(14)(15) \\
11(15), 1416,1576,148,162(17)\end{array}$ \\
\hline$[64,4]$ & 1 & 56 & $\begin{array}{l}1(14), 153(15), 169(16), 11(12)(16), 1157,19,1(12) 4,1427,124(11), 1144,12(16) 2 \\
1(16) 7,14(17) 9,12(11) 7,169(15), 1188\end{array}$ \\
\hline$[68,4]$ & 1 & 60 & $\begin{array}{l}14,1283,1642,15(17)(16), 1(16)(10), 1(11) 7,1(12) 29,12,1923,1287,17(15) 8,113 \\
12(14) 3,131(18), 17(10), 1582,149(12)\end{array}$ \\
\hline$[72,4]$ & 1 & 63 & $\begin{array}{l}14,15(14) 6,1(11) 9,1215,19(15), 15(17)(18), 1,168(18), 1278,11(17) 4,13(12) 8 \\
1(11) 7(15), 16(14)(15), 1192,164,1(11)(16) 6,111(12), 132\end{array}$ \\
\hline$[76,4]$ & 1 & 67 & $\begin{array}{l}1(10), 152(17), 1(12)(13), 113(17), 1(11) 4,12(13)(14), 1(11) 78,191(14), 191,13(12) 3 \\
14(12), 1358,1696,11(18)(15), 1(12) 2(15), 1(12) 8,1(18) 7,1194,1(13)(13)\end{array}$ \\
\hline$[80,4]$ & 1 & 71 & $\begin{array}{l}15,14(11) 4,12(18) 2,1(15) 9,1(13) 6(14), 129(14), 11(13)(16), 1153,12(14)(13), 128 \\
1(16)(17), 167(12), 12(10)(16), 13,11(11)(15), 14(14)(15), 12(17), 117(10), 1(12) 2(15) \\
14(18)(12)\end{array}$ \\
\hline$[88,4]$ & 1 & 78 & $\begin{array}{l}1(11), 1629,11(11)(15), 178(12), 14(17) 9,113(11), 12(18)(16), 12(13)(15), 1923 \\
113(12), 11(17), 14(15) 4,1287,125(13), 14(10) 5,102,1(12) 78,14(14)(17), 1(11) 53 \\
19(17), 154(17), 12(10)(12)\end{array}$ \\
\hline$[92,4]$ & 1 & 82 & $\begin{array}{l}1(12), 13(18)(14), 13(13) 6,1319,115(15), 106,164,12(17) 7,19(12) 7,14(14) 3,13(13) 3 \\
14(17) 9,19(12)(18), 112(17), 138(17), 1(11)(17), 1175,15(13)(12), 168,1(10) 58,181 \\
16(13) 3,178(12)\end{array}$ \\
\hline$[96,4]$ & 1 & 86 & $\begin{array}{l}17,1(11)(10) 8,18(17)(14), 1(18) 4,145(17), 13(17)(18), 1(11)(15), 12(14)(15), 1293 \\
11(16) 9,164(14), 1532,1(10) 78,12(10)(11), 14(13) 4,14(14) 2,186(14), 111(16) \\
15(13) 9,1569,132(17), 13,11(16)(14), 12(11)\end{array}$ \\
\hline$[100,4]$ & 1 & 89 & $\begin{array}{l}15,1,1(13) 9,131(15), 14(10) 7,1(10) 5(18), 11(16) 7,159(16), 16(14), 138(12), 11(18) 6 \\
11(11)(13), 118(10), 12(12) 7,115,19(11) 2,11(14)(15), 13,12(17) 4,1(10)(18), 17(18) 3 \\
17(10)(17), 15(12) 8,1(11) 78,141(12)\end{array}$ \\
\hline
\end{tabular}


Table 13. QT codes over $\mathbb{F}_{19}$ with $m=5$

\begin{tabular}{|c|c|c|c|}
\hline code & $\lambda$ & $d$ & $b_{i}(x)$ \\
\hline$[10,5]$ & 1 & $6^{*}$ & $104,1(10)(18) 59$ \\
\hline$[15,5]$ & 1 & $11^{*}$ & $114,11(15)(13)(10), 14(14)(10) 3$ \\
\hline$[20,5]$ & 1 & 15 & $105,11(15)(18)(12), 12(18)(15)(18), 131(12) 6$ \\
\hline$[25,5]$ & 1 & 19 & $12,117(10)(15), 198,12(12)(11)(13), 1(15)(14) 4$ \\
\hline$[30,5]$ & 1 & 23 & $11,11(15) 8(15), 11(16)(13) 9,1(11) 12,11634,1(16)(18) 8$ \\
\hline$[35,5]$ & 1 & 28 & $132(14)(15), 11,10(13)(18), 11(13)(11) 5,135(12)(15), 159(12), 1(14)(18) 1$ \\
\hline$[40,5]$ & 1 & 32 & $\begin{array}{l}1(13) 1,1185(15), 17637,16(13)(11) 8,1(15)(13) 2,131(14)(11), 17(12) 1(14) \\
1(18)(13)(14)\end{array}$ \\
\hline$[45,5]$ & 1 & 37 & $\begin{array}{l}1(12), 1(16)(10) 7,13(11)(15) 4,17(10) 53,1567(18), 12(17) 62,1(11) 71(15) \\
16(10) 8(17), 119(16) 3\end{array}$ \\
\hline$[50,5]$ & 1 & 41 & $\begin{array}{l}1(15)(15) 6,1298(11), 13(18) 4(14), 13,143(18) 9,1(14)(13)(11), 11766,1(13) 9(12) \\
124(10)(16), 19(13)(11) 2\end{array}$ \\
\hline$[55,5]$ & 1 & 46 & $\begin{array}{l}1(16), 14(13)(17)(12), 143(18)(11), 11(12) 7(10), 13(17)(16)(11), 12347,1(10)(15) 93 \\
138(12) 7,138(14)(17), 11(13)(11) 6,1(15)(11)\end{array}$ \\
\hline$[60,5]$ & 1 & 50 & $\begin{array}{l}1,15(12) 4(18), 11345,1562(15), 12145,12(10) 5(17), 108,18(16) 5,13(18) 8(12) \\
12(16)(12)(16), 15(18)(13)(15), 12634\end{array}$ \\
\hline$[65,5]$ & 1 & 55 & $\begin{array}{l}11(10), 11(12) 8(10), 16843,137(13) 4,18(14)(16) 6,1(10) 2,13(11)(10) 5,1237(15) \\
1287,17(18) 57,13(17) 95,13(15)(18) 8,13235\end{array}$ \\
\hline$[70,5]$ & 1 & 59 & $\begin{array}{l}13,13(11)(18) 8,1146(13), 11524,13739,12(10)(13)(16), 108(10), 16953,17(11)(17) \\
13(17) 74,1535,18387,16(13)(12)(11), 141(15)(12)\end{array}$ \\
\hline$[75,5]$ & 1 & 64 & $\begin{array}{l}13,1(13)(11)(13)(18), 12,13(16)(18) 4,189(13)(17), 12(18)(15), 1177(17), 145(13) \\
12(13)(12) 2,15(17)(16) 6,1013,11(17)(15)(11), 117(15), 11112,14(14) 57\end{array}$ \\
\hline$[80,5]$ & 1 & 68 & $\begin{array}{l}128(17) 3,1934(18), 18,132(10)(18), 184(14) 6,1(16) 52,133(16), 13(13)(15) 2 \\
12(15) 43,13(16) 17,14(14) 15,17586,12(15) 15,11962,17(14), 117(12) 9\end{array}$ \\
\hline$[85,5]$ & 1 & 73 & $\begin{array}{l}1(15), 13(16) 2,143(11) 9,12(16)(17) 4,1136(11), 13(18) 46,15(17) 59,1163(17), 1 \\
126(15) 6,13(16) 56,161(12) 8,10(16), 15(18) 5(18), 12(15)(16)(18), 135(14)(11) \\
17(17)(15)\end{array}$ \\
\hline$[90,5]$ & 1 & 77 & $\begin{array}{l}101,102,11(11)(11)(18), 1418(17), 1274,12(16) 7,13(17)(12)(18), 11(13)(16)(11) \\
1693,13(18)(14)(12), 15(17) 1(11), 11778,1(15) 1,13854,1457,11(14)(17) 4,1135 \\
12(13)(12)(14)\end{array}$ \\
\hline$[95,5]$ & 1 & 82 & $\begin{array}{l}1,12(14) 3(17), 101,11(17) 7(12), 14(17)(14)(15), 137(17)(16), 12(10) 49 \\
1(13)(14)(16), 12(14) 68,114,12(16) 1(12), 134(16) 5,18(12) 4,18(13) 2,161(15) 2 \\
1(10)(18)(11), 11(14) 4(14), 112(15)(14), 12(12) 43\end{array}$ \\
\hline$[100,5]$ & 1 & 87 & $\begin{array}{l}111,14,183(16), 192(13)(17), 17(17)(14) 6,11(15) 38,13(13)(18)(15), 13(18) 7(12) \\
1(12)(16) 1,13(13)(15) 5,1354(14), 164(18)(11), 1585,145(11) 5,1(11)(17) 4 \\
1(10)(18)(17), 11(15)(17)(11), 17(18) 7(18), 117(18)(12), 13(14) 46\end{array}$ \\
\hline$[105,5]$ & 1 & 91 & $\begin{array}{l}10(14), 11,14(12)(15), 1118(17), 12,1(13)(14) 3,18(14) 6(15), 14(13) 46,137(13) 9 \\
11(16)(17) 6,1247(12), 1354(16), 11(10) 27,115(14) 8,131(12) 3,12(18) 78,114(14) 2 \\
11(11) 75,1538,1(16)(16) 2,16(11) 4\end{array}$ \\
\hline$[110,5]$ & 1 & 96 & $\begin{array}{l}1025,14,18,193(10)(17), 12(10) 4(11), 1(10) 8(17)(14), 14(13) 2(18), 1(18)(18)(16) \\
137(10)(14), 11(18) 78,158(11)(15), 111(17) 6,1(13)(11)(13) 6,12(18) 42 \\
13(12)(16)(12), 11484,1383(15), 142(12) 6,12(15) 2(17), 11279,14(11)(16)(17) \\
1693(17)\end{array}$ \\
\hline$[115,5]$ & 1 & 100 & $\begin{array}{l}12,1(14) 5,1(11), 145(16) 5,131(14)(18), 11(16)(17)(10), 177(12), 198(13), 161(14) 6 \\
14(13)(10)(14), 181(13), 1239,11(13) 9(14), 18(11) 7(15), 12(10) 1(16), 13(12) 3(15) \\
167(12)(18), 11277,19(10) 1,1442,115(13)(17), 1(17)(10) 4,15(11)(12) 6\end{array}$ \\
\hline$[120,5]$ & 1 & 105 & $\begin{array}{l}118,1769(12), 1,142(10) 3,1(14)(17)(14), 12(12)(17)(18), 14(10) 8(17), 1616 \\
1529(16), 1(10)(17) 4(14), 1144(16), 135(12), 15(13) 4(14), 11853,1(12)(10)(17) \\
11(10), 153(13) 8,13(14) 34,18(14)(10)(14), 13(11) 1(18), 12(17) 5(14), 11(15)(17)(16) \\
1(17) 42,123(17)(16)\end{array}$ \\
\hline$[125,5]$ & 1 & 109 & $\begin{array}{l}102,17(16) 7,13894,13(15)(18)(14), 13(18)(10)(15), 12(14) 76,11,15696,142(13) 3 \\
11(11)(12) 9,15376,11(13) 76,115(10), 1(10) 52,112(17)(10), 1145(10), 18957 \\
132(11) 2,132(10) 6,118(11) 5,12(16), 13(14) 2(17), 13(13)(10) 3,15(18) 32 \\
134(11)(15)\end{array}$ \\
\hline
\end{tabular}

